\title{
Composición florística, estructura horizontal y potencial de conservación de árboles y arbustos de la Reserva Biológica Isla del Caño, Costa Rica.
}

\author{
Paul Eduardo Oviedo Pérez ${ }^{1}$ \\ ${ }^{1}$ Escuela de Ciencias Biológicas, Universidad Nacional, Heredia, Costa Rica; paul.oviedo.perez@est.una.ac.cr
}

Recibido: 25 de febrero de $2020 \quad$ Aceptado: 14 de Mayo de 2020

\section{RESUMEN}

El objetivo de este estudio fue analizar la composición florística, la estructura horizontal y el potencial de conservación de árboles y arbustos en Isla del Caño, Costa Rica. Se seleccionaron dos sitios con diferente estadio de sucesión (ladera y planicie). En cada sitio se establecieron siete parcelas $(10 \mathrm{~m}$ x $30 \mathrm{~m})$ y se realizó un inventario de todos los árboles con diámetro $\geq 10 \mathrm{~cm}$. En el centro de cada parcela se delimitó un cuadrante de $5 \mathrm{~m} \mathrm{x} 5 \mathrm{~m}$ y se identificaron los arbustos y árboles con diámetro menor a $10 \mathrm{~cm}$. La composición fue analizada a partir de las especies registradas y la cantidad de individuos por especie. La estructura horizontal fue analizada usando como parámetros la densidad arbórea, el índice de valor de importancia (IVI) y la distribución por clase diamétrica. Los resultados indicaron una diferencia significativa de la composición entre ambos sitios. El peso ecológico recae sobre las especies Brosimum utile y Ocotea insularis. Ambas especies se concentraron en la planicie; mientras que la vegetación de la ladera estuvo constituida por especies heliófitas. El porcentaje de similitud entre las especies de dosel y sotobosques fue de $17 \%$ en la ladera y $36 \%$ en la planicie. La regeneración de las especies de dosel fue abundante, principalmente para $B$. utile. La distribución de los árboles por clases diamétricas mostró la forma de "J" invertida. La mayoría de las especies fueron asignadas a una categoría de conservación baja. Este estudio confirma que pocas especies tuvieron alta dominancia y abundancia, posiblemente como respuesta de las especies a las condiciones ambientales de la isla. La abundancia de árboles y arbustos importantes para la restauración; así como, las especies maderables, justifican la conservación de la isla.

Palabras claves: bosque húmedo, comunidad vegetal, conservación, ecología forestal.

\footnotetext{
ABSTRACT

Floristic composition, horizontal structure and conservation potential of trees and shrubs on Isla del Caño, Biological Reserve, Costa Rica

The objective of this study was to analyze the floristic composition, the horizontal structure and the conservation potential of trees and shrubs on Isla del Caño, Costa Rica. Two sites with different stages of succession (slope and plain) were selected. At each site, seven plots were established $(10 \mathrm{~m}$ x $30 \mathrm{~m})$ and an inventory was made of all trees with a diameter $\geq$ $10 \mathrm{~cm}$. In the center of each plot a quadrant of $5 \mathrm{~m} \mathrm{x} 5 \mathrm{~m}$ was delimited and shrubs and trees with a diameter smaller than $10 \mathrm{~cm}$ were identified. The composition was analyzed from the registered species and the number of individuals per species. The horizontal structure was analyzed using the tree density, the importance value index (IVI) and the distribution by diametric class as parameters. The species identified were assigned to a conservation priority: urgent, high, medium, low. The results indicated a significant difference in the composition between both sites. The ecological weight falls on the species Brosimum utile and Ocotea insularis. Both species were concentrated in the plain; while the vegetation of the slope was constituted by heliophyte species. The percentage of similarity between canopy and understory species was $17 \%$ for the slope and $36 \%$ for the plain. Regeneration of the canopy species was abundant, mainly for B. utile. The distribution of the trees by diametric classes showed the inverted "J" shape. Most species were assigned to a low conservation category. This study confirms that few species had high dominance and abundance, possibly as a response of the species to the environmental conditions of the island. The abundance of trees and shrubs important for restoration; as well as, the timber species, justify the conservation of the island.
}

Key words: moist forest, plant community, conservation, forest ecology. 
La Isla del Caño fue declarada por el gobierno de Costa Rica como reserva biológica en 1978 (Boza \& Mendoza, 1980). Geológicamente es una de las zonas más antiguas del país y su edad está estimada entre 40 y 50 millones de años (Boza, 1988). Los historiadores coinciden en que la isla fue ocupada por la civilización indígena Chiriquis durante el periodo precolombino. Posteriormente, fue visitada por piratas y huaqueros en busca de objetos de valor arqueológico (Finch \& Honestschlager, 1986). Actualmente, el tráfico a nivel insular ha quedado restringido por el Sistema Nacional de Áreas de Conservación (SINAC).

La investigación biológica en la isla ha sido enfocada principalmente en el área marina (Guzmán, Cortés, Richmond \& Glynn, 1987; Guzmán, 1988; Guzmán \& Cortés, 1989; Salas, Sánchez-Godínez \& Montero-Cordero, 2015). Por el contrario, los inventarios en las 320 ha de la parte insular son escasos, a pesar de la importancia que conlleva el conocimiento científico de las especies terrestres para la conservación. Por ejemplo, los bosques son importantes porque mantienen la productividad del ambiente, proveen alimento y refugio para la fauna, estabilizan el ciclo del agua, contribuyen con la captura y almacenaje de carbono y reducen el calentamiento global (Sundquist et al 2008; Kuma \& Shibru, 2015).

El análisis de la composición florística y de la estructura vegetal constituye el primer paso para caracterizar la vegetación y comprender la dinámica de un bosque (Hartshorn \& Hammel, 1994; Cascante \& Estrada, 2001). Un análisis de la composición de una comunidad de plantas requiere la identificación de las especies y el número de individuos que se encuentran en un área determinada (Lamprecht, 1990; Oviedo, 2015); mientras que la estructura horizontal de un bosque es analizada con base a la distribución diamétrica, la densidad y dominancia de los individuos arbóreos (Hernández, 1999; Oviedo, Alvarado \& Fournier, 2009). Estos parámetros ecológicos ayudan a visualizar cómo los disturbios antropogénicos y naturales pueden afectar la vegetación de un área (Noumi, 2013; Neelo, Teketay, Kashe \& Masamba, 2015; Zhong et al. 2015; Sainge et al., 2019).

El potencial de conservación varía entre las especies, dependiendo de su distribución geográfica y de la probabilidad de extinción en un tiempo determinado (Londoño-Murcia \& Sánchez-Cordero, 2011). La Unión Internacional para la Conservación de la Naturaleza (UICN) clasifica a las especies en una determinada categoría de amenaza (situación crítica, amenazada y vulnerable), según sea la tasa de reducción de la población, la transformación del hábitat y el tamaño de la población (Mace et al., 2008). En este contexto, surge la creación de las áreas silvestres protegidas, como espacios esenciales para proteger y conservar la biodiversidad. No obstante, las áreas protegidas por sí solas no aseguran la conservación de una especie en particular, todo depende de si el lugar reúne los atributos de hábitat que la especie requiere para su establecimiento y perpetuación; así como, de la incorporación de las actividades sociales y económicas del desarrollo de la sociedad en las estrategias de manejo (Castaño, 2005; Toledo, 2005). El objetivo de este estudio fue analizar la comunidad arbórea y arbustiva del bosque de la Reserva Biológica Isla del Caño (RBIC) con base a la composición florística, la estructura horizontal y el potencial para la conservación de especies leñosas, de manera que se contribuya con la toma de decisiones para el manejo del bosque.

\section{Metodología}

Área de estudio: La Isla del Caño está ubicada aproximadamente a $15 \mathrm{~km}$ de Bahía Drake, Península de Osa y en la plataforma continental del Pacífico de Costa Rica $\left(08^{\circ} 42^{\prime} 4^{\prime}\right.$ 'N, 835'20”W). La topografía es irregular y está constituida de dos sitios claramente definidos. El primero consiste en un terreno inclinado (ladera a partir de ahora) que se extiende desde la línea costera hacia el interior de la isla. El segundo sitio está ubicado hacia el centro de la isla, donde el terreno es menos inclinado (planicie a partir de ahora) y alcanza elevaciones entre los 90 y 110 m.s.n.m. (Figura 1). Ambos sitios están cubiertos por un bosque siempre verde y claramente estratificado (Boza \& Cevo, 2002). El clima está caracterizado por una temperatura entre $26^{\circ} \mathrm{C}$ y $28^{\circ} \mathrm{C}$, una humedad relativa del $100 \%$, una precipitación media anual de 5150 mm y una estación seca de enero a abril (Chinchilla, Protti \& Cabrera, 2002). La zona de vida es bosque húmedo tropical (bh-T) (Holdridge, 1970). 

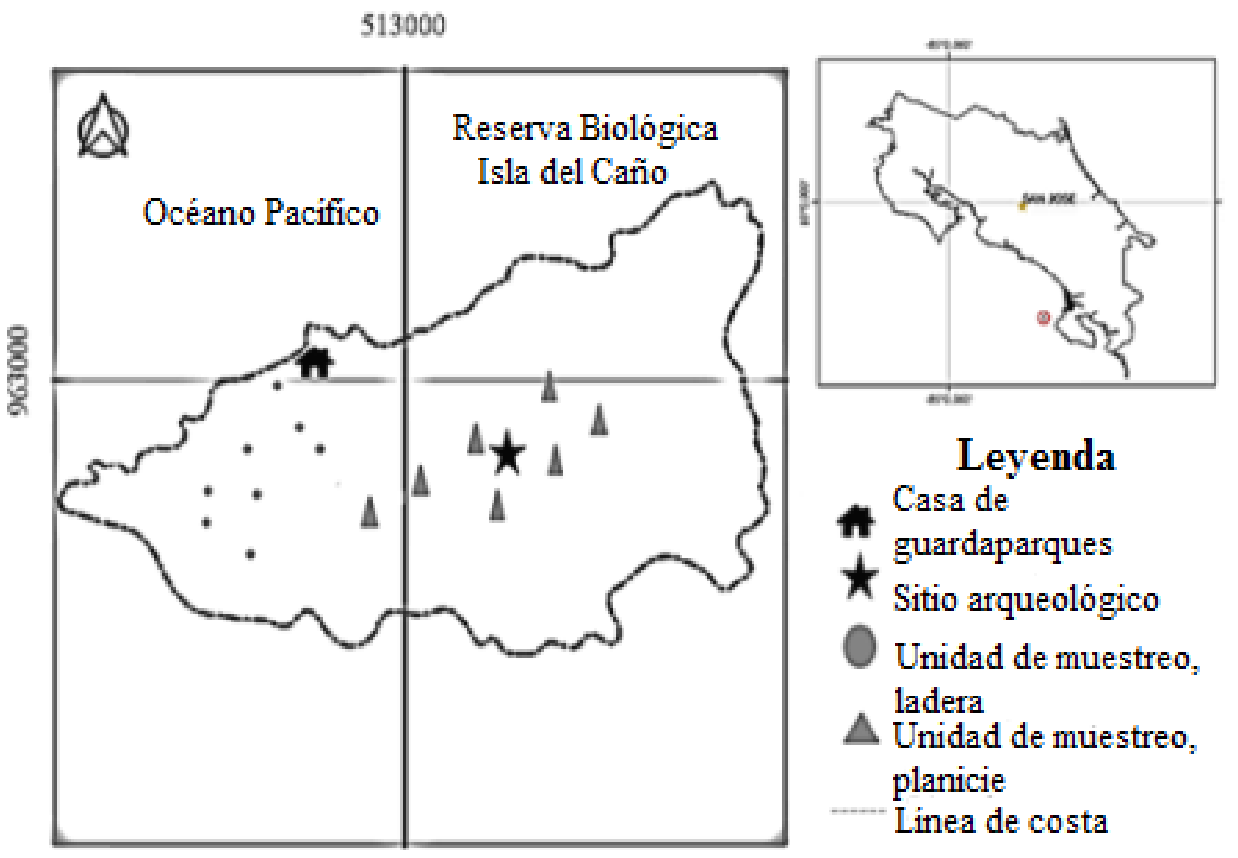

\section{Leyenda}

Casa de guardaparques

Sitio arqueológico

Unidad de muestreo, ladera

$\Delta$ Unidad de muestreo, planicie Linea de costa

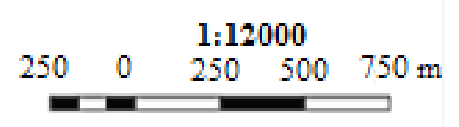

Fig. 1. Ubicación de la Isla del Caño, Área de Conservación Osa, Costa Rica.

Recolección de datos: la recolección de datos fue realizada en abril del 2019. Un total de 14 parcelas (10 m x $30 \mathrm{~m})$ fueron distribuidas aleatoriamente. Cada parcela fue dividida en tres subparcelas $(10 \mathrm{~m} \mathrm{x} 10 \mathrm{~m})$, siguiendo el método de Camacho (2000). Siete de estas parcelas fueron ubicadas en la ladera y otras siete en la planicie (Figura 1). La distancia mínima entre parcelas fue de $50 \mathrm{~m}$. Dentro de cada parcela se midió el diámetro $(\mathrm{cm})$ a 1,3 $\mathrm{m}$ de altura (DAP) de todos los árboles con un diámetro mayor o igual a $10 \mathrm{~cm}$. El diámetro $(\mathrm{cm})$ fue medido con una cinta diamétrica. Un cuadrante $(5 \mathrm{~m} \times 5 \mathrm{~m})$ fue establecido en el centro de cada parcela, para el inventario de los arbustos con un DAP menor a $10 \mathrm{~cm}$ y una altura superior a los $50 \mathrm{~cm}$, según la metodología sugerida por Gysel \& Lyon (1980).

La identificación taxonómica de cada árbol y arbusto se realizó in situ a nivel de familia, género y especie. Cuando la identificación no fue posible, se recolectó una muestra para su posterior identificación con manuales especializados (Holdridge \& Poveda, 1975; Quesada, Jiménez, Zamora, Aguilar \& González, 1997; Zamora, Jiménez \& Poveda, 2000; Zamora, Jiménez \& Poveda, 2004; Hammel, Grayum, Herrera \& Zamora, 2007; Jiménez, Rojas, Rojas \& Rodríguez, 2011); así como, la colaboración de expertos botánicos del Herbario Nacional y del Herbario Anastasio Alfaro González de la Escuela de Ciencias Biológicas, UNA. Las muestras recolectadas fueron preservadas y depositadas en el Herbario Anastasio Alfaro González.

Análisis de datos: la lista de especies registradas y sus respectivas abundancias fueron ordenadas en una matriz de comunidad. La comparación de la composición florística entre la ladera y la planicie fue realizada con la rutina no paramétrica ANOSIM ("analysis of similarities"), bajo la hipótesis nula de que no hay diferencia en la composición de especies entre ambos sitios de la isla. ANOSIM trabaja como una prueba de ANOVA, donde en lugar de operar sobre datos sin procesar, opera en una matriz de disimilitud clasificada (Clarke, 1993). Para evitar que las especies más comunes dominaran en el resultado final de la ordenación y para aumentar la influencia de las especies subordinadas en el modelo resultante, las abundancias fueron transformadas por medio de la función decostand y el método Hellinger del paquete estadístico vegan (Oksanen et al., 2013). La contribución de las especies a la diferenciación o similitud entre grupos fue analizada con el módulo SIMPER ("similarity profile"). La regeneración del bosque fue analizada por comparación de las especies presentes en el sotobosque con los árboles maduros, mediante el coeficiente de similitud de Jaccard (Chao, Chazdon, Colwell \& Shen, 2004).

La estructura horizontal fue analizada mediante un gráfico de distribución de los árboles por categorías diamétricas; asî como, por medio del valor de importancia (IVI) para cada especie. Los parámetros estructurales para la estimación del IVI fueron calculados utilizando las siguientes fórmulas: 
(1) Área basal de un árbol $=\pi(D A P / 2)^{2}$

(2) Densidad = número de individuos de la especie A/área muestreada.

(3) Densidad relativa = (número de individuos de la especie A/número total de individuos en el área) $\mathrm{x} 100$.

(4) Abundancia relativa = abundancia absoluta de la especie A/la abundancia absoluta de totas las especies.

(5) Frecuencia = número de parcelas en las cuales la especie A ocurre/número total de parcelas muestreadas.

(6) Frecuencia relativa = (valor de la frecuencia de la especie A/total de todos los valores de frecuencias de todas las especies) x 100.

(7) Dominancia = área basal de la especie A/área muestreada.

(8) Dominancia relativa $=($ dominancia para la especie $\mathrm{A} /$ dominancia total de todas las especies $) \times 100$.

(9) Índice de valor de importancia (IVI) = abundancia relativa + frecuencia relativa + dominancia relativa.

Las especies inventariadas fueron asignadas a una categoría de prioridad de conservación, utilizando como referencia los criterios de la lista roja de especies amenazadas (UICN, 2012; UICN, 2018), el decreto 25700 del Ministerio de Ambiente y Energía (MINAE) y la lista de especies maderables para Costa Rica (Jiménez et al., 2011; Moya, Rodríguez \& Olivares, 2014). Las categorías de prioridad de conservación establecidas para este estudio son:

- $\quad$ Urgente: especie considerada críticamente amenazada por la UICN y en peligro de extinción según MINAE.

- $\quad$ Alta: especie considerada como amenazada por la UICN y con población reducida o endémica según MINAE.

- Media: especie cuya población es vulnerable según la UICN o es maderable (Jiménez et al., 2011; Moya et al.,

2014).

- $\quad$ Baja: especie catalogada como de preocupación menor, pero que es importante para la restauración según los criterios de la UICN y no está registrada en la lista de especies amenazadas o vedadas según MINAE.

\section{Resultados}

Composición de especies. Un total de 820 individuos, distribuidos en 19 familias y 33 especies fueron identificados, siendo Melastomataceae la familia mejor representada con cinco especies, seguida por Fabaceae, Piperaceae y Rubiaceae, con tres especies respectivamente (Cuadro 1). Brosimum utile fue la especie arbórea más abundante, tanto en la ladera como en la planicie. La especie arbustiva más abundante fue Psychotria brachiata y estuvo distribuida en toda la isla.

Cuadro 1. Lista de especies y distribución espacial $(\mathrm{L}=$ ladera, $P=$ planicie $)$ de árboles $\mathrm{y}$ arbustos registrados en la Reserva Biológica Isla del Caño, Puntarenas, 2019.

Familia/Nombre científico

Nombre vernáculo

Acanthaceae

Acanthaceae

Bignoniaceae

Amphitecna latifolia (Mill.) A.H.Gentry

Malvaceae

Bombacopsis sessilis (Benth.) Pittier

Pseudobombax septenatum (Jacq.) Dugand

Clusiaceae

Calophyllum brasiliense Cambess.

Calophyllum longifolium Willd

Erythroxylaceae

Erythroxylum macrophyllum Cav.

Euphorbiaceae

Alchornea costarricensis Pax \& K.Hoffm.

Fabaceae

Cojoba sp. B

Inga bella M.Sousa

Swartzia simplex (Sw.) Spreng.

Hypericaceae

Vismia baccifera (L.) Planch. \& Triana

Lacistemataceae 


\begin{tabular}{|c|c|c|c|}
\hline Lacistema aggregatum (P.J.Bergius) Rusby & Cafecillo, colpachí & $\mathrm{x}$ & $\mathrm{x}$ \\
\hline \multicolumn{4}{|l|}{ Lauraceae } \\
\hline Ocotea insularis (Meisn.) Mez & Aguacatón & $\mathrm{x}$ & $\mathrm{x}$ \\
\hline \multicolumn{4}{|l|}{ Malvaceae } \\
\hline Herrania purpurea (Pittier) R.E. Schult & Cacao de mono & $\mathrm{x}$ & $\mathrm{x}$ \\
\hline \multicolumn{4}{|l|}{ Melastomataceae } \\
\hline Clidemia capitellata (Bonpl.) D. Don & & $\mathrm{x}$ & \\
\hline Miconia appendiculata Triana & & & $\mathrm{x}$ \\
\hline Miconia argentea (Sw.) DC. & Cainillo, santa maría & $\mathrm{x}$ & \\
\hline Miconia oinochrophylla Donn. Sm. & & $\mathrm{x}$ & $\mathrm{x}$ \\
\hline Miconia prasina (Sw.) DC. & Camasey blanco & & $\mathrm{x}$ \\
\hline \multicolumn{4}{|l|}{ Moraceae } \\
\hline Brosimum utile (Kunth) Oken & Vaco, lechoso & $\mathrm{x}$ & $\mathrm{x}$ \\
\hline Ficus maxima Mill. & Higuerón & $\mathrm{x}$ & \\
\hline \multicolumn{4}{|l|}{ Phyllanthaceae } \\
\hline Hieronyma alchorneoides Allemão & Pilón, nancitón & $\mathrm{x}$ & \\
\hline \multicolumn{4}{|l|}{ Piperaceae } \\
\hline Piper aequale Vahl & & $\mathrm{x}$ & $\mathrm{x}$ \\
\hline Piper reticulatum $\mathrm{L}$. & & & $\mathrm{x}$ \\
\hline \multicolumn{4}{|l|}{ Piper corrugatum Kuntze. } \\
\hline \multicolumn{4}{|l|}{ Rubiaceae } \\
\hline Palicourea triphylla DC & & $\mathrm{x}$ & \\
\hline Pentagonia tinajita Seem. & & $\mathrm{x}$ & $\mathrm{x}$ \\
\hline Psychotria brachiata Sw. & & $\mathrm{x}$ & $\mathrm{x}$ \\
\hline \multicolumn{4}{|l|}{ Salicaceae } \\
\hline Casearia aculeata Jacq. & & $\mathrm{x}$ & \\
\hline Casearia sylvestris $\mathrm{Sw}$. & & $\mathrm{x}$ & \\
\hline \multicolumn{4}{|l|}{ Urticaceae } \\
\hline Cecropia obtusifolia Bertol & Guarumo & $\mathrm{x}$ & \\
\hline \multicolumn{4}{|l|}{ Verbenaceae } \\
\hline Citharexylum caudatum L. & Dama, huelenoche & $\mathrm{x}$ & \\
\hline
\end{tabular}

El análisis de varianza aplicado (ANOSIM) mostró una diferencia significativa de la composición florística de especies arbóreas entre los sitios ladera y planicie $\left(\mathrm{F}_{(1,13)}=4,8056 ; P=0,002\right)$. Los análisis SIMPER mostraron que $B$. utile y Ocotea insularis fueron las especies responsables del $51 \%$ de las disimilitudes de la composición entre los sitios (Cuadro 2). El porcentaje de similitud entre especies de dosel y sotobosque fue de 17\% para la ladera y 36\% para la planicie (Cuadro 3 ).

Cuadro 2. Contribución de las especies a las diferencias (disimilitud) entre sitios (ladera vs planicie) basado en análisis SIMPER

\begin{tabular}{lccc}
\hline \multicolumn{1}{c}{ Especie } & Media & $\begin{array}{c}\text { Desviación } \\
\text { estándar }\end{array}$ & $\begin{array}{c}\text { Contribución media por } \\
\text { especie }\end{array}$ \\
\hline Brosimum utile & 0,247159 & 0,13256 & 0,3277 \\
\hline Ocotea insularis & 0,139268 & 0,12792 & 0,5124 \\
\hline Lacistema aggregatum & 0,089885 & 0,08609 & 0,6315 \\
\hline Calophyllum brasiliense & 0,079385 & 0,07412 & 0,7368 \\
\hline Hieronyma alchorneoides & 0,064760 & 0,04191 & 0,8367 \\
\hline Alchornea costaricensis & 0,025601 & 0,03792 & 0,8796 \\
\hline Ficus maxima & 0,017516 & 0,02829 & 0,9016 \\
\hline Casearia aculeata & 0,016433 & 0,04091 & 0,9234 \\
\hline Bombacopsis sessilis & 0,016433 & 0,04091 & 0,9379 \\
\hline Calophyllum longifolium & 0,010934 & 0,02750 & 0,9521 \\
\hline Cecropia obtusifolia & 0,010714 & 0,02678 & 0,9644 \\
\hline Casearia sylvestris & 0,009299 & 0,02319 & 0,9768 \\
\hline Inga bella M.Sousa & 0,009299 & 0,02319 & 0,9891 \\
\hline Miconia argentea & 0,009299 & 0,02319 & 1,0000 \\
\hline Posoqueria latifolia & 0,008217 & 0,02046 & \\
\hline
\end{tabular}


Estructura horizontal. La densidad de especies arbóreas fue de $757 \pm 139,9$ individuos $^{-1}{ }^{-1}$ (media \pm desviación estándar). B. utile tuvo la densidad más alta (300 individuos ha ${ }^{-1}$ ); mientras que Inga bella fue la especie arbórea con la menor densidad (2,38 individuos $\left.\mathrm{ha}^{-1}\right)$. La especie arbórea con mayor frecuencia fue $B$. utile, seguida por $O$. insularis e Hieronyma alchorneoides. La especie encontrada como más dominante y con mayor IVI fue B. utile $(71,41 \%$ y $125,14 \%$, respectivamente); mientras que las especies con IVI menor a 5\% fueron Cecropia obtusifolia, I. bella y Posoqueria latifolia (Cuadro 4). La distribución de los árboles por categorías diamétricas demostró una estructura discetánea en forma de "J" invertida, donde los árboles con menor diámetro del tallo se ubicaron en las primeras clases diamétricas (Fig. 2).

Potencial de conservación. Aproximadamente un $77 \%$ de las especies fueron catalogadas como de preocupación menor, tanto a nivel nacional como regional. Asimismo, la mayoría de las especies son consideradas importantes para la restauración del bosque. El segundo grupo en importancia para la conservación estuvo constituido por especies de categoría media. Dos especies de leguminosas (I. bella y Cojoba sp. B) fueron las únicas en la categoría de prioridad de conservación alta. No hubo registros de especies consideras en la categoría urgente (Fig. 3).

Cuadro 3.Similitud florística de la vegetación de dosel y sotobosque

\begin{tabular}{|c|c|c|}
\hline \multicolumn{2}{|c|}{ Especies exclusivas } & \multirow{2}{*}{$\frac{\text { Especies compartidas }}{\text { Sotobosque-Dosel }}$} \\
\hline Sotobosque & Dosel & \\
\hline Amphitecna latifolia & Alchornea costarricensis & Brosimun utile \\
\hline Citharexylum caudatum & Bombacopsis sessiles & Calophyllum brasilience \\
\hline Clidemia capitellata & Calophyllum longifolium & Casearia aculeata \\
\hline Erythroxylum macrophyllum & Casearia sylvestris & Ocotea insularis \\
\hline Herrania purpurea & Cecropia obtusifolia & Lacistema aggregatum \\
\hline Miconia appendiculata & Hieronyma alchorneoides & Posoqueria latifolia \\
\hline Miconia argentea & Inga bella & \\
\hline Miconia oinochrophylla & Ficus maxima & \\
\hline \multicolumn{3}{|l|}{ Miconia prasina } \\
\hline \multicolumn{3}{|l|}{ Palicourea triphylla } \\
\hline \multicolumn{3}{|l|}{ Pentagonia tinajita } \\
\hline \multicolumn{3}{|l|}{ Piper aequale } \\
\hline \multicolumn{3}{|l|}{ Piper reticulatum } \\
\hline \multicolumn{3}{|l|}{ Piper corrugatum } \\
\hline \multicolumn{3}{|l|}{$\begin{array}{l}\text { Pseudobombax septenatum } \\
\text { Psychotria brachiata }\end{array}$} \\
\hline \multicolumn{3}{|l|}{ Cojoba sp. B } \\
\hline \multicolumn{3}{|l|}{ Ruellia tonduzii } \\
\hline \multicolumn{3}{|l|}{ Swartzia simplex } \\
\hline Vismia baccifera & & \\
\hline
\end{tabular}

Cuadro 4. Estructura horizontal basado en el índice de valor de importancia (IVI) de las especies arbóreas, Isla del

\begin{tabular}{|c|c|c|c|c|}
\hline Nombre científico & $\begin{array}{c}\mathbf{A r} \\
(\%)\end{array}$ & $\begin{array}{l}\mathbf{F r} \\
(\%)\end{array}$ & $\begin{array}{c}\text { Dr } \\
(\%)\end{array}$ & IVI \\
\hline Alchornea costaricensis Pax \& K.Hoffm. & 4,61 & 6,28 & 1,61 & 12,5 \\
\hline Bombacopsis sessilis (Benth.) Pittier & 1,54 & 4,18 & 2,82 & 8,54 \\
\hline Brosimum utile (Kunth) Pittier & 32,32 & 21,22 & 71,41 & 125,14 \\
\hline Calophyllum brasiliense Cambess. & 10,77 & 8,67 & 1,48 & 20,92 \\
\hline Calophyllum longifolium Willd. & 0,77 & 2,12 & 0,11 & 3,0 \\
\hline Casearia aculeata Jacq. & 6,16 & 2,12 & 0,42 & 8,7 \\
\hline Cecropia obtusifolia Bertol. & 1,54 & 2,12 & 0,34 & 4,0 \\
\hline Hieronyma alchornoides Allemao & 9,25 & 14,95 & 5,07 & 29,25 \\
\hline Inga bella $\mathrm{M}$. Sausa & 0,77 & 2,12 & 1,49 & 4,38 \\
\hline Ficus maxima Mill. & 0,14 & 4,18 & 2,35 & 8,07 \\
\hline Lacistema aggregatum (P. J. Bergius) Rusby & 16,15 & 12,85 & 2,6 & 28,52 \\
\hline Ocotea insularis (Meisn.) Mez & 16,15 & 17,07 & 10,21 & 43,51 \\
\hline Posoqueria latifolia (Rudge) Roem. \& Schult. & 1,54 & 2,12 & 0,08 & 3,74 \\
\hline
\end{tabular}




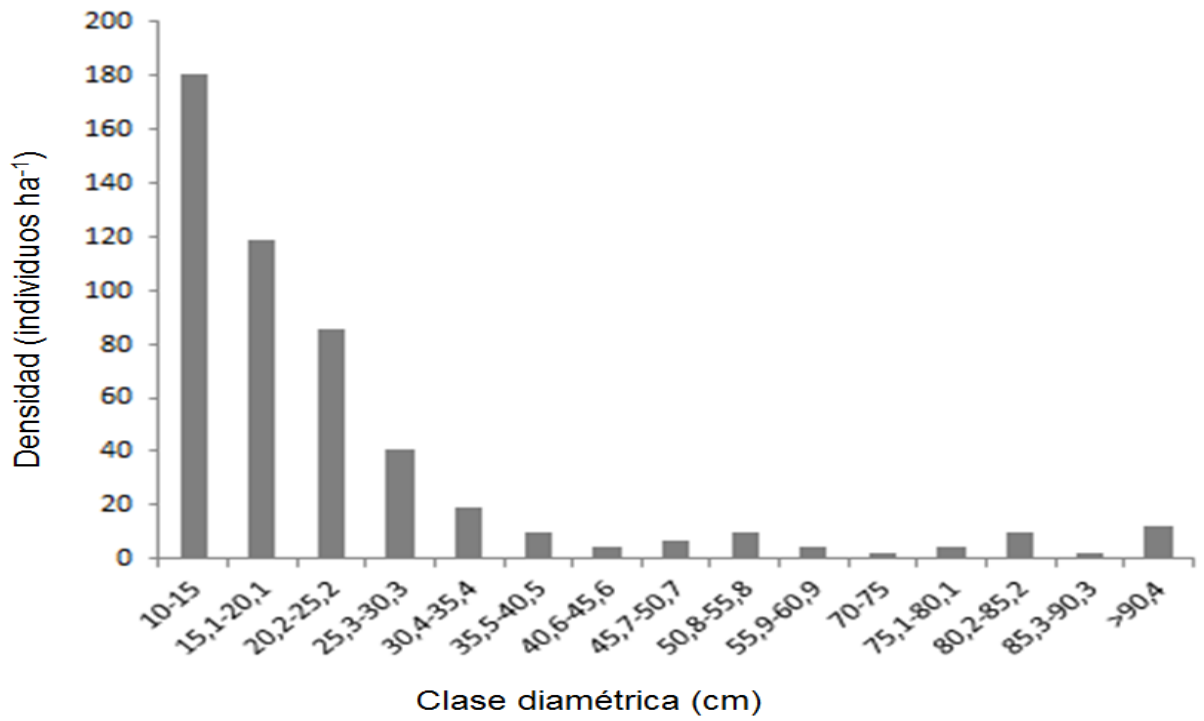

Fig. 2. Distribución de árboles por clases diamétricas, Isla del Caño, Puntarenas, Costa Rica, 2019.

\section{Discusión}

La cantidad de especies vegetales (árboles y arbustos) de Isla del Caño es menor en comparación con otros bosques húmedos tropicales ubicados a nivel continental en la costa del Pacífico de Costa Rica (Morales et al., 2012; Oviedo, 2015), pero tiene una mayor riqueza si se compara con otras islas del país (Vargas \& Hidalgo, 2013; Porras-Jiménez, Acosta-Vargas, Castillo \& Quesada-Monge, 2014). Además, la lista de especies puede ser mayor si se realizara un inventario de aquellas especies que se han adaptado a las condiciones más áridas y a alta salinidad de la línea costera. La composición florística de un bosque está directamente relacionada con la precipitación anual; no obstante, los eventos históricos de perturbación, la latitud, la biología de las especies y las interacciones planta-animal, son otros factores que influyen en el ensamblaje de la comunidad vegetal (Asquith, 2002; Guariguata \& Ostertag, 2002). Un cambio en la composición florística entre la ladera y la planicie indica diferentes estados de sucesión entre ambos sitios. El terreno inclinado de la ladera podría favorecer condiciones más iluminadas en los distintos estratos del bosque (Vieira \& Scariot, 2006) y, por ende, una mayor competencia de especies heliófitas efímeras (Alchornea costaricensis, Miconia argentea y Psycotria brachiata) y heliófitas durables (Hieronyma alchornoides, Casearia spp. y Lacistema agreggatum) (Morales et al. 2012). Por el contrario, en la planicie se ubicaron los árboles con tallas diamétricas más grandes (DAP $>50 \mathrm{~cm}$ ), por lo que el dosel es más continuo y se crea un sotobosque amplio (Ellsworth \& Reich, 1993).

La cantidad de árboles por hectárea fue similar a la densidad arbórea reportada para bosques húmedos tropicales con un estadio de sucesión avanzado (Oviedo et al., 2009, Morales et al., 2012). Tanto los resultados del análisis SIMPER como del IVI muestran que el peso ecológico recae sobre las especies $B$. utile y $O$. insularis. Por consiguiente, ambas especies juegan un papel importante en la dinámica del bosque; además, son las principales responsables de cerrar el dosel y retener la humedad en el sotobosque. Por otro lado, el hecho de que la comunidad forestal esté dominada por especies esciófitas, indica una asociación tardía y que las especies han utilizado al máximo la capacidad del sitio (Porras-Jiménez et al., 2014). Las pendientes leves a medias y los suelos arcillosos son las condiciones ambientales idóneas para B. utile (Jiménez et al., 2011). Esta especie también se caracteriza por su regeneración abundante alrededor del árbol madre, lo cual garantiza el flujo de árboles en el futuro y mantiene la estructura del bosque establecido a lo largo del tiempo y espacio (Palacios, Palacios \& Abadia, 2015).

La estructura diamétrica discetánea (modelo "J" invertida) es típica de bosques primarios intervenidos y no intervenidos; así como, de bosques secundarios en avanzado estado de sucesión (Louman, Valerio \& Jiménez, 2001). Una concentración de individuos en las clases diamétricas menores se da en respuesta a procesos de competencia y a la adaptación de las especies a las condiciones lumínicas y ambientales del bosque; además indica que pocas especies alcanzan grandes tamaños (Porras-Jiménez et al. 2014).

La prioridad de conservación baja que obtuvo la mayoría de las especies vegetales indica que la isla protege pocas especies amenazadas, al menos a nivel insular. No obstante, este grupo de especies tiene un valor ecológico agregado. Por un lado, proveen recurso alimenticio para la avifauna (e.g. Cecropia obtusifolia, Ficus maxima, Lacistema aggregatum y Miconia argentea). Por otra parte, participan en el control de la erosión del suelo, estabilizan los cauces fluviales y restauran las 
áreas degradas (UICN, 2018). Además, es importante destacar el valor que tiene este bosque en la conservación de especies consideradas como maderables, entre ellas: B. utile, H. alchornoides y Calophyllum brasiliense.

La planicie es el sitio en estadio de sucesión más avanzado y, por lo tanto, es la zona más conservada de la isla. Los disturbios en esta parte del bosque deben responder a los objetivos de manejo de una reserva biológica, restringiendo el ingreso únicamente con propósitos de investigación. Por el contrario, el tráfico de turistas por el sendero que recorre la ladera puede ser regulado, previo a un estudio de capacidad de capacidad de carga, una adecuada interpretación ambiental y la capacitación de guías turísticos certificados.

En conclusión, la composición florística entre la ladera y la planicie en Isla del Caño están en diferente estadio de sucesión. Hacia la ladera se ubican las especies heliófitas de árboles y arbustos; mientras que en la planicie se incrementa la dominancia de B. utile y $O$. insularis. La regeneración de especies de dosel es abundante, especialmente en aquellas especies con un mayor IVI. La distribución discetánea indica una mayor reserva de los árboles en las primeras clases diamétricas, por lo que se asegura el equilibrio del bosque. La parte insular de esta reserva biológica es importante para la conservación de especies maderables. El ingreso de personas a la planicie debe estar restringido a la investigación, debido al avanzado estadio de sucesión; mientras que la ladera tiene un alto potencial turístico, pero requieren un adecuado plan de manejo.

\section{Agradecimientos}

Agradezco a Costa Rica Dive and Surf, la Perla del Sur y Fundación Keto Costa Rica por facilitar el transporte hacia la isla. Al Ministerio de Ambiente y Energía y al Área de Conservación OSA por brindar los permisos de investigación. A Fabián Araya Yannarella, Rolando Calderón Fallas, Esteban Salazar Acuña y Alonso Quesada por su apoyo en la identificación de las especies y la revisión científica del manuscrito. A José Guillermo Ramírez Bogantes por la elaboración del mapa de la isla.

\section{Referencias}

Asquith, N. (2002). La dinámica del bosque y la diversidad arbórea (pp. 377-406). In: Guariguata, M. R., \& Kattan, G. H. (Eds), Biología y conservación de bosques tropicales. Cartago, Costa Rica: LUR.

Boza, M. A. (1988). Parques Nacionales de Costa Rica. San José, Costa Rica: Heliconia.

Boza, M. A., \& Cevo, J. A. (2002). Parques nacionales y otras áreas protegidas de Costa Rica. San José, Costa Rica: INCAFO.

Boza, M., \& Mendoza, R. (1980). Los parques nacionales de Costa Rica. Madrid, España: INCAFO.

Camacho, M. (2000). Parcelas permanentes de muestreo en bosque natural tropical. Turrialba, Costa Rica: CATIE.

Castaño, G. (2005). Áreas silvestres protegidas, criterios para su selección y problemáticas $\quad$ en su conservación. Boletín científico. Museo de Historia Natural, 10, 79-101.

Chao, A., Chazdon, R. L., Cowell, R. K., \& Shen, T. (2004). A statistical method for the in species composition with incidence and abundance data. Ecology Letters, 8, 148-159.

evaluation of similarity

Clarke, K. R. (1993). Non-parametric multivariate analyses of changes in community structure. Austral Ecology, 18(1), $117-143$.

Condit, R., Pérez, R., \& Daguerre, N. (2011). Trees of Panama and Costa Rica. New Jersey, $\quad$ EE. $\quad$ UU: Princeton University Press.

Cascante, A., \& Estrada, A. (2001). Composición florística y estructura de un bosque húmedo premontano en el Valle Central de Costa Rica. Revista de Biología Tropical, 49(1), 213-225.

Chinchilla, I., Protti, M., \& Cabrera, J. (2002). Peces dulceacuícolas de la Isla del Caño: biogeográficos. UNICIENCIA, 19, 53-57.

distribución y aspectos

Ellsworth, D. S., \& Reich, P. B. (1993). Canopy structure and vertical patterns of traits in a deciduous forest. Oecologia, 96(2), 169- 178.

photosynthesis and related leaf

Finch, W. O., \& Honestschlager, K. (1986). Preliminary archeological research on Isla del

Caño. Journal of the 
Steward Anthropological Society, 14(1-2), 189-203.

Guariguata, M. R., \& Ostertag, R. (2002). Sucesión secundaria (pp. 591-624). In: ～Guariguata, M. R., \& Kattan, G. H. (Eds). Biología y conservación de bosques neotropicales. Cartago, Costa Rica: LUR.

Guzmán, H. M. (1988). Distribución y abundancia de organismos coralívoros en los arrecifes coralinos de la Isla del Caño, Costa Rica. Revista de Biología Tropical, 36, 191-207.

Guzmán, H. M., \& Cortés, J. (1989). Coral reef community structure at Caño Island, Pacific $\quad$ Costa Rica. P.S.Z.N.I. Marine Ecology, 10, 23-41.

Guzmán, H. M., Cortés, J., Richmond, R. H., \& Glynn, P. W. (1987). Efecto del fenómeno “El Niño Oscilación Sureña" 1982/83 en los arrecifes coralinos de la Isla del Caño, Costa Rica. Revista de Biología Tropical, $35,325-332$.

Gysel, L., \& Lyon, L. (1980). Análisis y evaluación de hábitat. In: Rodríguez, R. (Ed), Manejo y gestión de la vida silvestre. EE. UU: WWF//Fish and Wildlife Service.

Hammel, B. F., Grayum, M. H., Herrera, C., \& Zamora, N. (2007). Manual de Plantas de $\quad$ Costa Rica. Dicotiledóneas. Vol V-VIII. EE. UU: Missouri Botanical Garden Press/INBio/Museo Nacional de Costa Rica.

Hartshorn, G. S., \& Hammel, E. (1994). Vegetation and floristic patterns (pp.73-89). In: $\quad$ McDade, L., Bawa K., Hespenheide, H., \& Hartshorn, G. (Eds), La Selva Ecology and Natural History of a Neotropical Rain Forest. Chicago: University of Chicago Press.

Hernández, Z. T. (1999). Cronosecuencias del bosque seco tropical en el Parque Nacional Palo Verde, Bagaces, Costa Rica. Práctica de especialidad. Cartago, Costa Rica: Instituto Tecnológico de Costa Rica.

Holdridge, L. R. (1970). Life zone ecology. San José, CR: Tropical Science Center.

Holdridge, L. R., \& Poveda, L. J. (1975). Árboles de Costa Rica. Vol. I. San José, Costa ～Rica: CCT.

Jiménez, Q., Rojas, F., Rojas, V., \& Rodríguez, L. (2011). Árboles maderables de Costa $\quad$ Rica. $\quad$ Ecología y silvicultura. Santo Domingo, Heredia, Costa Rica: INBio.

Kuma, M., \& Shibru, S. (2015). Floristic composition, vegetation structure, and regeneration status of woody plant species of Oda forest of Humbo Carbon Project, Wolaita, Ethiopia. Journal of Botany, 2015, 1-9.

Londoño-Murcia, M. C., \& Sánchez-Cordero, V. (2011). Distribución y conservación de especies amenazadas en Mesoamérica, Chocó y Andes tropicales. Revista Mexicana de Biodiversidad, 82, 926-950.

Louman, B., Valerio, J., \& Jiménez, W. (2001). Bases ecológicas (pp. 21-75). In: Louman, $\quad$ B., Quirós, D., \& Nilson, M. (Eds), Silvicultura de bosques latifoliados húmedos con énfasis en América Central. Turrialba, Costa Rica: CATIE.

Mace, G. M., N. J. Collar, K. J. Gaston, C. Hilton-Taylor, H. R. Akçakaya, N. Leader- Williams, E. J. Milner-Gulland \& Stuart. S. N. (2008). Quantification of extinction risk: IUCN's system for classifying threatened species. Conservation Biology 22:1424-1442.

Morales, M., Vílquez, B., Chazdon, R. L., Ortega, M., Ortiz, E., \& Guevara M. (2012). Diversidad y estructura horizontal en los bosques tropicales del Corredor Biológico de Osa. Revista Forestal Mesoamericana Kurú, 9(23), 19-28.

Moya, R., Rodríguez, A., \& Olivares, C. (2014). Árboles maderables de la Península de Osa: madera y corteza. Cartago, Costa Rica: Editorial Tecnológica de Costa Rica.

Sainge, M.N., Lyonga, N.M., Mbatchou, G.P.T., Kenfack, D., Nchu, F., \& Peterson, A.T. ～～(2019). Vegetation, floristic composition and structure of a tropical montane forest in Cameroon. Bothalia, 49(1), 1-13.

Neelo, J., Teketay, D., Kashe, K., \& Masamba, W. (2015). Stand structure, diversity and regeneration status of 
woody species in open and enclosed dry woodland sites around Molapo farming area of the Okavango delta, northeastern Botswana. Open Journal of Forestry 5, 313-328.

Noumi, E. (2013). Floristic inventory of woody species in the Manengouba Mountains Forest Cameroon. Journal of Biology and Life Science 4, 282-309.

Oksanen, J., Blanchet, F. G., Kindt, R., Legendre, P., Michin, P. R., O’Hara, R. B., S Simpson, G. L., Solymos, M., Stevens, R. H., \& Wagner, E. (2013). Community Ecology Package (Package vegan). Disponible en: http://vegan.r-forge.r- project.org/

Oviedo, P. E. (2015). Composición y estructura horizontal de la Reserva Absoluta de Cabo Blanco y remanentes de bosque del sector de Cabuya, Puntarenas, Costa Rica. Repertorio Científico, 18(2), 95-102.

Oviedo, P. E., Alvarado, A., \& Fournier, L. (2009). Caracterización de la vegetación alrededor de los senderos del sector Quebrada González, Parque Nacional Braulio $\quad$ Carrillo, Costa Rica. UNICIENCIA 23, 41-49.

Palacios, L., Palacios, J., \& Abadia, D. (2015). Densidad poblacional de Brosimum utile en un bosque con actividades de minería y tala en el Chocó, Colombia. Cuadernos de Investigación, 7(2), 319-323.

Porras-Jiménez, M., Acosta-Vargas, L., Castillo, M., \& Quesada-Monge, R. (2014). $\quad$ Estructura y composición florística del bosque nuboso de la Isla de Coco. Tecnología en Marcha. VI Encuentro de Investigación y Extensión, 22-36.

Quesada, F., Jiménez, Q., Zamora, N., Aguilar, R., \& González, J. (1999). Árboles de la $\quad$ Península de Osa. Santo Domingo, Heredia, Costa Rica: INBio.

Salas, E., Sánchez-Godínez, C., \& Montero-Cordero, A. (2015). Peces marinos de la Reserva Biológica Isla del Caño: Estructura de las comunidades de peces de arrecife y lista taxonómica actualizada de los peces costeros. Revista de Biología Tropical, 63 (Suppl. 1), 97-116.

Sundquist, E., Robert, B., Stephen, F., et al. (2008). Carbon sequestration to mitigate climate change. N. Y., EE. UU: USGS.

Toledo, V. (2005). Repensar la conservación ¿áreas naturales protegidas o estrategia $\quad$ bioregional. Gaceta Ecológica, 77, 67-83.

UICN. (2012). Directrices para el uso de los Criterios de la Lista Roja de la UICN a nivel Versión 4.0. Gland, Suiza y Cambridge, Reino Unido: UICN.

regional y nacional:

UICN. (2018). The UICN Red List of Threatened Species. Recuperado de <http://www.iucnredlist.org>

Vargas, G., \& Hidalgo, J. E. (2013). Sucesión de un bosque tropical seco en la Isla San Lucas, Puntarenas, Costa Rica. Cuadernos de Investigación, 5(2), 261-269.

Vieira, D. L., \& Scariot, A. (2006). Principles of Natural Regeneration of Tropical Dry Forests $\quad$ for Restoration. Restoration Ecology, 14(1), 11-20.

Zamora, N., Jiménez, Q., \& Poveda, J. L. (2000). Árboles de Costa Rica. Vol. II. Heredia: ～Editorial INBio/CCT/CI.

Zamora, N., Jiménez, Q., \& Poveda, J. L. (2004). Árboles de Costa Rica. Vol III. Heredia: $\quad$ Editorial INBio/CCT/CI.

Zhong, L., Chang-Yang, C., Lu, P., Gu, X., Lei, Z., Cai, Y., Zhen, F., Sun, I., \& Yu, M. (2015). Community structure and species composition of the secondary evergreen broad-leaved forest: The analyses for a 9 ha forest dynamics plot in Wuyanling N Nature Reserve, Zhejiang Province, East China. Biodiversity Science 23, 619-629. 
APENDICE I. Algunas especies vegetales de la Reserva Biológica Isla del Caño, Puntarenas, Costa Rica; A - B) Ocotea insularis (rama y gambas), C) Calophyllum brasiliense, D - E) Brosimum utile (rama, corteza y savia), F) Psychotria brachiata, G) Lacistema aggregatum, H) Cojoba sp. B, I) Erythroxylum macrophyllum (Fotografía: Paul E. Oviedo).

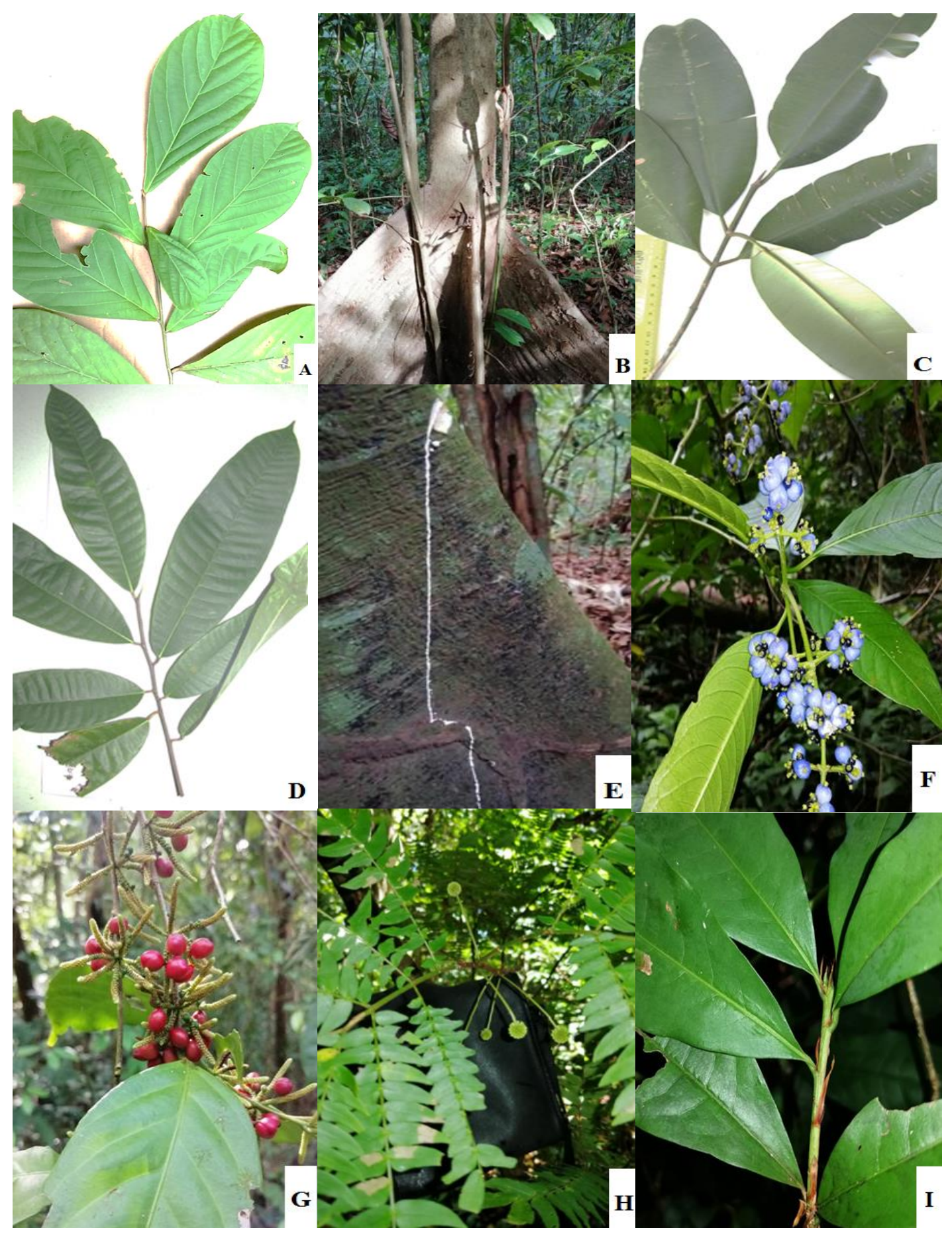

